\title{
High fidelity audio signal reconstruction method based on multi channel
}

\author{
negative sequence current
}

\author{
CHEN Yubo
}

Beijing university of posts and Telecommunications, China, 100876

Keywords: negative-sequence current; multi-channel; sound signal; reconstitution

\begin{abstract}
Signal processing method is one of important technological bases in the existing fault diagnosis of mechanical equipment, and the degree of accuracy of analysis results is the key factor for whether the diagnosis is successful. Research on spectral analysis is one of the existing main development directions. The digital signal processing is basically to solve signal processing problems from two aspects, including that: the first is time domain method (namely digital filtering; the second is frequency domain method (namely spectral analysis). The paper has mainly introduced discrete Fourier transform and fast Fourier transform; made an in-depth research and study form foundation, and mastered the key of FFT algorithm by researching DFT and FFT algorithms. With the study of DSP chip operating principle and development environment, the sample commissioning of CCS and software simulation have been grasped, and the real-time spectral analysis for signal on the DSP clip is realized.
\end{abstract}

\section{Introduction}

In the digital signal processing, the discrete Fourier transform (Discrete. Time Fourier Transform, DFT) is the frequently-used transform method, and it plays an important role in the digital information processing system. The frequency discretization discovered by discrete Fourier transform may directly be used for analyzing signal frequency spectrum and frequency response of counter filter and realizing convolution operation of signal through line system, etc; therefore, it has great effect in the aspect of spectral analysis of signal. For too heavy computation burden of DFT, it is still difficult for problems to make real-time processing even though computer is adopted. Therefore, with unremitting efforts made by many scholars, the universal fast Fourier transform (FFT) appears. Fast Fourier transform (Fast Fourier Transform, FFT) is not another transform different form discrete Fourier transform, and is a kind of fast and effective algorithm to reduce DFT calculation frequency. Researches on FFT algorithm and its realization method have great meaning. At present, FFT has been widely used in many fields of spectral analysis, matched filtering, digital communication, image processing, automatic speech recognition, radar processing, remote sensing and metering, geological prospecting and wireless secrecy communication, etc. FFT processors with different performance requirements are required in different application sites. It is necessary for FFT processor to have performances of high speed, high accuracy, large capacity and real-time processing in many application fields. Therefore, how to faster and more flexibly realize FFT has become more and more important. 


\section{Modeling of high fidelity audio signal based on multi-channel}

In the collection and transmitting process of sound, because of limitation of equipment and device, the response of amplitude to frequency is not consistent, and it is highly possible that the gain of some frequency components is too great or too small. With regard to such defect of frequency response characteristic, appropriate adjustment is required. For equalization algorithm of audio signal, it is promoted or attenuated by adjusting the gain of some certain frequency components with software design. Acoustical signal may make up for defect of frequency response characteristic after equalization processing, which could also artificially create some preferable audio effect.

Audio signal (for example: the acoustical signal of electric guitar is $150 \mathrm{mV}$ electrical signal) obtains a string of digital signals after ADC conversion with high precision and high speed, and they are input to the buffer RAM under framing. Then, with manual control of one or several processing algorithm(s), tune audio signal in the inside of TMS320C5402 for high-speed computation. Audio signals processed are later input to DAC converter with high precision and high speed to restore simulated acoustical signals, and they are amplified and output by power amplifier of voice box. By taking advantage of multi-channel buffer asynchronous serial port (McBSP) of TMS320VC5402 and PCM 1800 and PCM1744 clip interfaces, the collection and output of audio signal are realized. The purpose of using buffer is to make real-time processing with sound effect. Each module in system is processed at the same time. A part of signals are being converted in ADC; while another part of signals are simultaneously making algorithm processing in DSP processor, which means that the overall system is operated in the form of production line. Experiments show that: the hardware and software system designed based on DSP is an excellent system for collection and processing of audio signal.

PCM1744 is the two-channel stereo DAC, containing digital filter and output amplifier, with dynamic range of $95 \mathrm{~dB}$ and various sample frequencies for selection (the maximum is $96 \mathrm{kHz}$ ). Adopt 24-bitIIS data input format. Operation of PCM1744 mainly involves several pins with requirements for time sequence including LRCIN (sample clock signal input), BCKIN (bit clock signal input), SCKI (system clock input) and DIN (data input). After connection between PCM1744 and "C5402, "5402 uses buffer serial port 1 to send data; various clock signals are generated by "C5402, and PCM 1744 passively receives all kinds of information. System clock signal (SCKI) of PCM1744 is provided by TOUT pin of "C5402, and TOUT is the pin of output signal of timer of "C5402 which has relatively strong drive capability and may drive several clips. The format of timer for data acceptance of PCM1744 must be IIS format, and "C5402 must satisfy requirements of IIS format when setting various kinds of clock modes in the register of buffer serial port.

\section{Signal reconstruction algorithm}

Fast Fourier transform (FFT) is the fast algorithm of discrete Fourier transform; it is obtained from improvement of algorithm of discrete Fourier transform according to characteristics of being odd, even, imaginary and real, etc of discrete Fourier transform, and it has no new discovery for discrete Fourier transformer.

Finite length sequence $\mathrm{x}(\mathrm{n})$ and its frequency domain expression $\mathrm{X}(\mathrm{k})$ may be obtained from the following discrete Fourier transform 


$$
\begin{array}{r}
\mathrm{x}(\mathrm{k})=\operatorname{DFT}[\mathrm{x}(\mathrm{n})]=\sum_{n=0}^{N-1} x(n) W_{N}^{n k} \quad 0 \leq k \leq N-1 \\
\mathrm{x}(\mathrm{n})=\operatorname{IDFT}[\mathrm{X}(\mathrm{k})]=\frac{1}{N} \sum_{n=0}^{N-1} X(k) W_{N}^{-n k} \quad 0 \leq k \leq N-1
\end{array}
$$

The formula (1) is called as discrete Fourier unitary transform; while the formula (2) is called as discrete Fourier inverse transform, and $\mathrm{x}(\mathrm{n})$ and $\mathrm{X}(\mathrm{k})$ constitute discrete Fourier transform pair.

According to the above formula, for calculation of a $\mathrm{X}(\mathrm{k})$, the complex multiplication for $\mathrm{N}$ time(s) and complex addition for $\mathrm{N}-1$ time(s) are required; while for calculation of all $\mathrm{X}(\mathrm{k})$ ( $0 \leq k \leq N-1$ ), the complex multiplication for $N^{2}$ time(s) and complex addition for N(N-1) are required. To realize complex multiplication once, the real number multiplication for four times and real number addition twice are required. When $\mathrm{N}$ is relatively great, for real-time signal processing, there are completely strict requirements for computation speed of processor; therefore, the problem on how to reduce calculation of discrete Fourier transform is of great important.

To reduce computation burden and improve operating rate, the algorithm must be improved. In the coefficient needing to be calculated in the calculation process of DFT, there are quite a few symmetries existing. Through research on such symmetry, the calculation may be simplified in the calculation process, so that time required for DFT may be reduced.

As what mentioned before, the frequency of complex multiplication of DFT of $\mathrm{N}$ point is equal to $N^{2}$. Obviously, with DFT of $\mathrm{N}$ point divided into several shorter DFT, the frequency of multiplication is greatly reduced. In addition, the twiddle factor $W_{N}^{m}$ has obvious periodicity and symmetry, and the periodicity is:

$$
W_{N}^{m+l N}=e^{-j \frac{2 \pi}{N}(m+l N)}=e^{-j \frac{2 \pi}{N} m}=W_{N}^{m}
$$

Its symmetry is presented as:

$$
W_{N}^{-m}=W_{N}^{N-m} \text { 或 }\left[W_{N}^{N-m}\right]^{*}=W_{N}^{m} \quad W_{N}^{-m}=W_{N}^{N-m} \text { or }\left[W_{N}^{N-m}\right]^{*}=W_{N}^{m}
$$

FFT algorithm is to constantly divide DFT with long sequence into several DFT(s) with short sequence, and reduce frequency of calculation of DFT by taking advantage of periodicity and symmetry of $W_{N}^{m}$.

$W_{N}^{n k}$ has the following inherent characteristics:

(1) Periodicity of $W_{N}^{n k}: W_{N}^{n k}=W_{N}^{(n+N) k}=W_{N}^{n(k+N)}$

(2) Symmetry of $W_{N}^{n k}: W_{N}^{-n k}=\left(W_{N}^{n k}\right)^{n}=W_{N}^{n(N-k)}$

(3) Reducibility of $W_{N}^{n k}: W_{N}^{n}=W_{N / n}, W_{N}=W_{N n}^{n}$

In addition, $W_{N}^{N / 2}=-1, W_{N}^{(k+N / 2)}=-W_{N}^{k}$ 
By taking advantage of the above characteristics of $W_{N}^{n k}, \mathrm{x}(\mathrm{n})$ or $\mathrm{X}(\mathrm{k})$ sequence is divided into short sequence as a certain rule for calculation, which may avoid a great number of repetitive calculation and improve calculation speed of DFT in such manner. Algorithm pattern has many kinds, but is basically divided into two kinds, including FFT algorithm with decimation in time (Decimation In Time, DIT) and FFT algorithm with decimation in frequency (Decimation In Frequency, DIF).

\section{Conclusion}

The system for collection and processing of audio signal designed in the paper has the following algorithms, including: compressing module, distortion module, ZNR/AMP module, equalization module, modulation module and delayed reverberation module. Each module may be used both separately and in series, and two LED digital displays are adopted to express mixed sound effect mode that has been well selected. It greatly changes its own timbre, and may generate various kinds of separate timbre effects of being compressed, distorted, frog voice, room noise generated (ZNR), amplified, equalization, chorus, cording, and delayed echo, etc. Several kinds of timbre effects may also simultaneously be used, which could greatly enrich performance effect in site. Same signals are respectively input into ZOOM 505 and this system; then, output waveforms on time domain and frequency domain, amplitude and phase are separately analyzed and compared for optimized processing of algorithm, and it can be found that the final timbre effect has not much different. In addition, in such system, there is both $\mathrm{A} / \mathrm{D}$ and $\mathrm{D} / \mathrm{A}$, which constitutes a closed loop with capability of self-transmitting and self-receiving; however, algorithms are concentrated in DSP chip for modularized processing, which brings great convenience to design and commissioning of system. Therefore, if the sound effector of electric guitar with function and effect compared to those of ZOOM 505 is designed based on proposal taking TMS320VC5402 DSP chip as a system for collection and processing of audio signal in the paper, the situation where national existing market [3] of sound effector of electric guitar is basically monopolized by foreign production will be fundamentally changed, which has very strong and practical value.

\section{Reference}

[1] Talebi N, Sadrnia M A, Darabi A. Robust Fault Detection of Wind Energy Conversion Systems Based on Dynamic Neural Networks[J]. Computational Intelligence \& Neuroscience, 2014, 10(2014):230-235.

[2] Prathosh A P, Ananthapadmanabha T V, Ramakrishnan A G. Epoch Extraction Based on Integrated Linear Prediction Residual Using Plosion Index[J]. IEEE Transactions on Audio Speech \& Language Processing, 2013, 21(12):2471-2480.

[3] Wang Y, Shi P, Li K, et al. An energy efficient medium access control protocol for target tracking based on dynamic convey tree collaboration in wireless sensor networks[J]. International Journal of Communication Systems, 2012, 25(9):1139-1159.

[4] Talebi N, Sadrnia M A, Darabi A. Robust Fault Detection of Wind Energy Conversion Systems Based on Dynamic Neural Networks[J]. Computational Intelligence \& Neuroscience, 2014, 10(2014):230-235. 